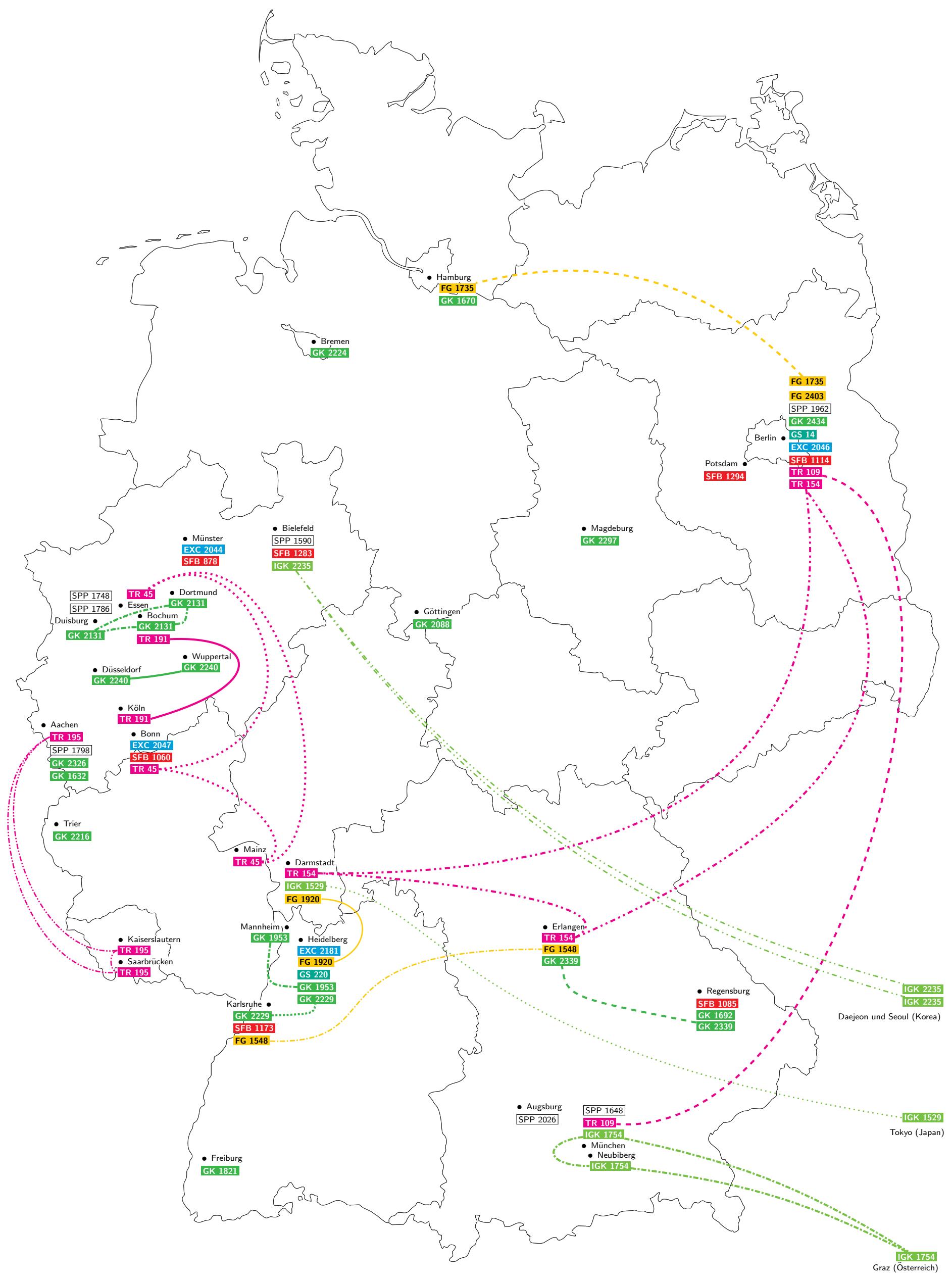




\section{Forschungsgruppe 1548}

Geometry and Physics of Spatial Random Systems

Karlsruhe, Erlangen

Forschungsgruppe 1735

Structural Inference in Statistics: Adaptation and

Efficiency

Berlin, Hamburg

\section{Forschungsgruppe 1920}

Symmetrie, Geometrie und Arithmetik

Heidelberg, Darmstadt

\section{Forschungsgruppe 2402}

Rough Paths, Stochastic Partial Differential

Equations and Related Topics

Berlin

\section{SPP 1590}

Probabilistic Structures in Evolution

Bielefeld

SPP 1648

Software for Exascale Computing

München

SPP ${ }_{1748}$

Reliable Simulation Techniques in Solid Mechanics.

Development of Non-standard Discretization

Methods, Mechanical and Mathematical Analysis

Duisburg-Essen

SPP 1786

Homotopietheorie und algebraische Geometrie

(Duisburg-)Essen

SPP 1962

Nichtglatte Systeme und Komplementaritätsprobleme

mit verteilten Parametern: Simulation und

mehrstufige Optimierung

Berlin

SPP 1798

Compressed Sensing in Information Processing

(CoSIP)

Aachen

SPP 2026

Geometrie im Unendlichen

Augsburg

\section{SFB 878}

Gruppen, Geometrie und Aktionen

Münster

\section{SFB 1060}

Die Mathematik der emergenten Effekte

Bonn

\section{SFB 1085}

Höhere Invarianten - Wechselwirkungen zwischen Globaler Analysis und Arithmetischer Geometrie

Regensburg

\section{SFB 1114}

Skalenkaskaden in komplexen Systemen

Berlin

\section{SFB 1173}

Wellenphänomene: Analysis und Numerik

Karlsruhe

\section{SFB 1283}

Unsicherheit beherrschen und Zufall sowi

Unordnung nutzen in Analysis, Stochastik und deren

Anwendungen

Bielefeld

\section{SFB 1294}

Datenassimilation: Die nahtlose Verschmelzung von Daten und Modellen

Potsdam

\section{Transregio 45}

Perioden, Modulräume und Arithmetik algebraischer Varietäten

Mainz, Bonn, Essen

Transregio 109

Diskretisierung in Geometrie und Dynamik

Berlin, München

Transregio 154

Mathematische Modellierung, Simulation und Optimierung am Beispiel von Gasnetzwerken

Erlangen, Berlin, Darmstadt

Transregio 191

Symplektische Strukturen in Geometrie, Algebra und Dynamik

Köln, Bochum

\section{Transregio 195}

Symbolische Werkzeuge in der Mathematik und ihre Anwendung

Kaiserslautern, Aachen, Saarbrücken

\section{Exzellenzcluster 2044}

Mathematik Münster: Dynamik - Geometrie -

Struktur

Münster

Exzellenzcluster 2046

$\mathrm{MATH}^{+}:$Forschungszentrum der Berliner

Mathematik

Berlin

\section{Exzellenzcluster 2047}

Hausdorff Center for Mathematics: Grundlagen, Modelle, Anwendungen

Bonn

\section{Exzellenzcluster 2181}

STRUKTUREN: Emergenz in Natur, Mathematik und komplexen Daten

Heidelberg

\section{GK 1632}

Experimentelle und konstruktive Algebra Aachen

\section{GK 1670}

Mathematics inspired by string theory and QFT

Hamburg

GK 1692

Curvature, Cycles, and Cohomology

Regensburg

GK 1821

Kohomologische Methoden in der Geometrie Freiburg

\section{GK 1953}

Statistische Modellierung komplexer Systeme und

Prozesse - Moderne nichtparametrische Ansätze

Mannheim, Heidelberg

GK 2088

Strukturerkennung in komplexen Daten:

Zusammenspiel von Statistik, Optimierung und inversen Problemen

Göttingen

\section{GK 2126}

Algorithmic Optimization (ALOP)

Trier
GK 2131

Phänomene hoher Dimensionen in der Stochastik-

Fluktuationen und Diskontinuität

Bochum, Dortmund, Duisburg

\section{GK 2224}

$\mathrm{Pi}^{3}:$ Parameter Identification - Analysis, Algorithms, Implementations

Bremen

\section{GK 2229}

Asymptotische Invarianten und Limiten von Gruppen und Räumen

Karlsruhe, Heidelberg

\section{GK 2240}

Algebro-geometrische Methoden in Algebra,

Arithmetik und Topologie

Düsseldorf, Wuppertal

GK 2297

Mathematische Komplexitätsreduktion Magdeburg

\section{GK 2326}

Energie, Entropie und Dissipative Dynamik

Aachen

\section{GK 2339}

IntComSin: Grenzflächen, komplexe Strukturen und singuläre Grenzwerte in der KontinuumsmechanikAnalysis und Numerik

Regensburg, Erlangen

\section{GK 2433}

Differentialgleichungs- und Daten-basierte Modelle in den Lebenswissenschaften und der Fluiddynamik (DAEDALUS)

Berlin

\section{GK 2434}

Facetten der Komplexität

Berlin

\section{Internationales Graduiertenkolleg 1520}

Mathematical Fluid Dynamics

Darmstadt, Tokyo

\section{Internationales Graduiertenkolleg 1754}

Optimierung und Numerik für partielle

Differentialgleichungen mit nichtglatten Strukturen

München, Neubiberg, Graz

Internationales Graduiertenkolleg 2235

Das Reguläre im Irregulären: Analysis von singulären und zufälligen Systemen

Bielefeld, Daejeon, Seoul

\section{Graduiertenschule 14}

Berlin Mathematical School

Berlin

Heidelberger Graduiertenschule der mathematischen und computergestützten Methoden für die

Wissenschaften

Heidelberg

\footnotetext{
Abkürzungen

EXC Exzellenzcluster

FG Forschungsgruppe

GK Graduiertenkolleg

GS

IGK Internationales Graduiertenkolleg

SFB Sonderforschungsbereich

SPP Schwerpunktprogramm

TR Transregio
} 